\title{
Two theorems about electromagnetic force in activate anisotropic regions
}

\author{
Dariusz Spałek
}

\begin{abstract}
The paper has dealt with two problems of calculation of electromagnetic force/torque. The first one is for magnetically anisotropic and conductive region. It has been presented sufficient condition for surface-integral representation of electromagnetic force/torque in conductive and anisotropic region. The second approach deals with the problem of independence of force/torque calculated value from shape of integral-surface. The second theorem gives the sufficient condition for this independence for Maxwell stress tensor method is applied.
\end{abstract}

Index Terms -- anisotropy, forced/active anisotropy, electromagnetic torque, solid rotor motor.

\section{ELECTROMAGNETIC FIELD FORCES}

The electromagnetic field forces [1,2] theoretical analysis is still vital problem $[3,4,5,6]$. For evaluation of the electromagnetic force density is applied as follows

$$
\vec{f}=\vec{f}_{L}+\vec{N}+\vec{M}
$$

which is proved (see [12], Appendix). The proof bases on Lorentz force density formula, Maxwells equations, and assumptions that displacement current (Poynting force [3]) and magnetic polarization (hysteresis force) can be neglected. In Eqn (1) $\vec{f}_{\mathrm{L}}$ denotes Lorentz force density, $\overrightarrow{\mathrm{N}}$ is the so-called nonhomegenity component [12] (it appears in nonhomogeneous regions), and

$$
\vec{M}=\frac{1}{2}\left(v_{v u}-v_{u v}\right) B_{v} \operatorname{grad}\left(B_{u}\right)
$$

is the anisotropy component for magnetic field.

Total electromagnetic force/torque can be calculated by the following equation

$$
\vec{f}=-\vec{i}_{u} d i v_{u}\left(\vec{\sigma}_{u}\right)-\vec{\Delta}
$$

where Maxwell's stress appears [12].

\section{THE FIRST THEOREM - SURFACE-INTEGRAL REPRESENTATION FOR MAGNETICALLY ANISOTROPIC AND CONDUCTIVE REGION}

$\mathrm{T}$

HE first theorem considers the equivalence between both volume and surface integrals representations for total electromagnetic force

/torque. This problem could be called as force/torque surface-integral representation problem.

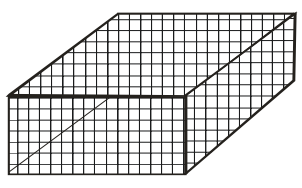

volume integral

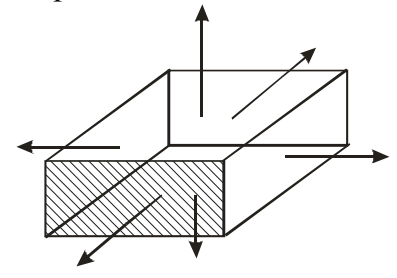

surface integral
Fig. 1. Equivalence of volume and surface integrals

This problem is analogous to the surface-integral

Dariusz Spalek is with the Department of Electrical Engineering Faculty of Silesian University of Technology in Poland representation of total electric charge placed in finite volume due to Gaussian law. The next analogous problem appears for Ampere law. The contour-integral representation of total currents passing through the surface is considered. It is known $[1,2,4,5,6,11]$ that the surface-integral representation for electromagnetic field forces can be introduced for electromagnetic field regions if the Maxwell stress tensor is symmetrical. The symmetry of the Maxwell stress tensor is guaranteed for isotropic media, only [1,2]. The main interest is whether for some anisotropic media the surface-integral of force/torque representation is tenable. The answer is positive under an extended condition. The theorem of surface integral representation is satisfied for either isotropic or anisotropic media (normal anisotropy). For isotropic medium the reluctivity (permeability) matrix is diagonal and all pivot values are equal to each other. The normal anisotropy is stated for the media which reluctivity (permeability) matrix is symmetrical one. There are also media for those the reluctivity (permeability) matrix is asymmetrical (the socalled active media [8]). It will be proved that

for magnetic field where the following conditions are satisfied

- no nonhomogeneous force(no reluctance force),

- no hysteresis phenomenon appears,

the force calculated by Lorentz's (volume integral) and Maxwell (surface integral) methods lead to the same result for magnetically anisotropic region if reluctivity matrix is symmetrical i.e. for

$u \neq v$ it is satisfied

$$
v_{v u}=v_{u v}
$$

If the condition (4) is satisfied thus the anisotropy component vanishes $M_{\mathrm{u}}=0$ - due to Eqn (2). It should be pointed out that if the condition (4) is not satisfied

$$
v_{v u} \neq v_{u v}
$$

the surface-integral representation is not possible, generally. The mathematical proof of this theorem is based on Eqn (1). Indeed, the assumptions specified above and Eqn (1) for $\mathrm{u}^{\text {th }}$ force component lead to the relation as follows

$$
f_{u}=f_{L u}+M_{u}=-\operatorname{div}_{u}\left(\vec{\sigma}_{u}\right)-\Delta_{u}
$$

For properly chosen co-ordinate system it can be set $\Delta_{u}=0$ (e.g. for Cartesian co-ordinate system always $\Delta_{u}=0$, for cylindrical system $\Delta_{\alpha}=0$; see Appendix), thus according to (3) is can be written

$$
L_{u} f_{u}=L_{u} f_{L u}=-\operatorname{div}\left(\vec{\sigma}_{u}\right)
$$

where $\mathrm{L}_{\mathrm{u}}$ means lame coefficient. Applying Gaussian theorem is obtained the formula

$$
\int_{V} L_{u} f_{u} d V=-\int_{S} \vec{\sigma}_{u} d \vec{S}
$$

that proves the thesis. This means that for regions with symmetric reluctivity matrix (either isotropic or normal 
anisotropic region) the Lorentz force/torque can be presented by surface-integral of the Maxwell stress tensor. In other words, the surface-integral representation is possible for media with symmetric reluctivity matrix. The above statement, however it applies only to a small range group of anisotropic materials, is important from theoretical point of view. In order to explain this theorem in physical way electromechanical converter with solid cylindrical rotor has been considered. An example confirms this thesis for asymmetrical stress tensor for simple electromechanical converter containing anisotropic (example). The analysis of electromagnetic field has been provided with the help of variable separation method. This way of analysis is chosen for giving precise insight into torque calculations.

\section{THE SECOND THEOREM - SURFACE-INTEGRAL REPRESENTATION FOR MAGNETICALLY ANISOTROPIC AND NONCONDUCTIVE REGION}

The surface-integral representation of electro-magnetic force/torque it is important problem from both theoretical and computational point of view. Let us regard electromagnetic force/torque that is exerted in finite region $\mathrm{R}$ (e.g. electromechanical converter moving part - rotor, carriage) can be evaluated by surface integral over the surface $S$ (e.g. either $S_{1}$ or $S_{2} \neq S_{1}$ ). The surface $S$ must surround the whole finite region $\mathrm{R}$. If the outer region $\mathrm{R}_{\text {out }}$ does not involve electromagnetic force/torque thus the surface $S$ (e.g. $S_{1}$ or $S_{2} \neq S_{1}$ ) can be placed differently in the gap fulfilling condition that it must surrounds whole exerting force region R. Exemplary, for electromechanical converter such as rotating electric machine the electromagnetic torque does not depend on the radius of surface $S$ placed in the gap region - Fig.2. The independence of the force/torque surface integral results from magnetic feature of the gap - it is the air-gap (i.e. the vacuum gap).

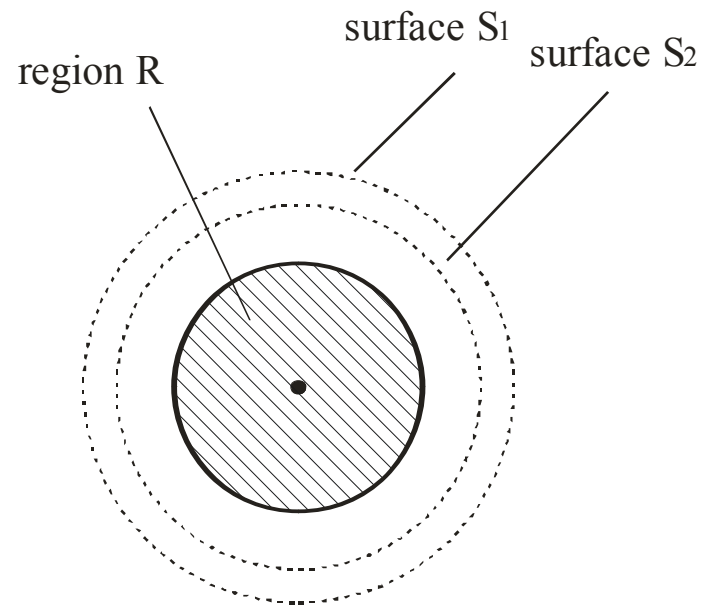

Fig. 2. The electromagnetic torque evaluation by surface integral - crosssection

The problem affects to electrostatic while calculating total electric charge with the help of Gauss law. The outer surface (Gaussian surface) must be spread so as to surround the whole charge independently of its shape, but the surrounding region must not contain any charge. The gap which surrounds the active region $\mathrm{R}$ (e.g. rotor) could be filled with ferrofluid $[9,10]$. As a consequence, the outer region $R_{\text {out }}$ (e.g. the gap) could be either isotropic or anisotropic. The second theorem affects the condition for possibility of surface-integral representation for different magnetic features of surrounding region $\mathrm{R}_{\text {out }}$ (e.g. the gap). Let us consider problem of calculation of electromagnetic force/torque acting on region $\mathrm{R}$. The region $\mathrm{R}$ affecting electromagnetic force/torque is included in volume $\mathrm{V}$. The volume $\mathrm{V}$ can be presented as a sum of

$$
V=R \oplus(V / R)
$$

where the $V / R=R_{\text {out }}$ denotes the region which surrounds active region $R$. The integrals that lead to total electromagnetic force and torque values exerted in region $\mathrm{R}$

$$
\vec{F}_{V}=\int_{V} \vec{f} d V=\int_{R} \vec{f} d V+\int_{R_{\text {out }}} \vec{f} d V
$$

and

$$
\vec{T}_{V}=\int_{V}(\vec{r} \times \vec{f}) d V=\int_{R}(\vec{r} \times \vec{f}) d V+\int_{R_{\text {out }}}(\vec{r} \times \vec{f}) d V
$$

respectively. The total electromagnetic force and torque are affected in region $\mathrm{R}$, thus

$$
\vec{F}_{V}=\vec{F}_{e}+\int_{R_{\text {out }}} \vec{f} d V=\vec{F}_{e}+\Delta \vec{F}_{\text {out }}
$$

and

$$
\vec{T}_{V}=\vec{T}_{e}+\int_{R_{\text {out }}}(\vec{r} \times \vec{f}) d V=\vec{T}_{e}+\Delta \vec{T}_{\text {out }}
$$

where $\vec{F}_{V}, \vec{T}_{V}$ are force and torque calculated over the volume $\mathrm{V} ; \vec{F}_{e}, \vec{T}_{e}$ are total electromagnetic force and torque; $\Delta \vec{F}_{\text {out }}, \Delta \vec{T}_{\text {out }}$ denote the residual integrals over the outer region $\mathrm{R}_{\text {out }}$. Now, the theoretical problem can be formulated precisely. Whether the electromagnetic force/torque arising in region $\mathrm{R}$ can be calculated by surface-integral over the volume constant?

Practical meaning of this question is whether the electromagnetic force/torque can be calculated by surfaceintegral over different surfaces which includes the experiencing force/torque region $\mathrm{R}$. If the answer is positive the total electromagnetic force/torque can be calculated for any surface which surrounds $\mathrm{R}$ (e.g. for different boundary surfaces $S_{1}$ and $S_{2}$ as shown in Fig. 3).

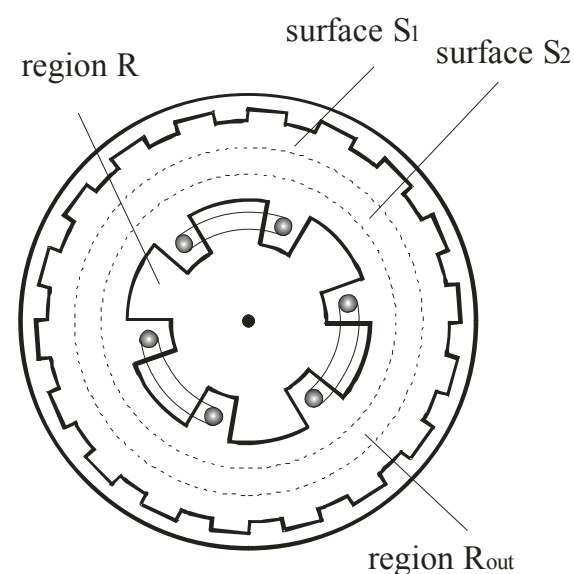

Fig. 3. The electromagnetic torque does not depend on radius of integralsurface in air-gap

The solution of the problem results from Eqns (1), (2). If for the region $\mathrm{R}_{\text {out }}$ is assumed, that: it is nonconductive, frequency is small (no displacement current), region is homogeneous, and there is no hysteresis phenomenon, hence only anisotropic component can give a contribution to residual integrals in Eqns $(11 \mathrm{a}, \mathrm{b})$. In other words, only 
magnetic anisotropy of the gap do not affect on electromagnetic force/torque, because the anisotropy component can be different from zero if for $\mathrm{u} \neq \mathrm{v}$ it is

$$
\boldsymbol{V}_{v u \delta}=\boldsymbol{V}_{u v \delta}
$$

Now, it could be formulated the second theorem

The electromagnetic force/ torque value calculated with the help of either volume integral or Maxwell stress tensor surface integral does not depend on surface position in the gap if the condition (12) is satisfied.

\section{ELECTROMAGNETIC TORQUE CALCULATION - EXAMPLE}

In order to confirm the theoretical results described above, an example of electromechanical converter with solid conductive rotor is considered Fig4.
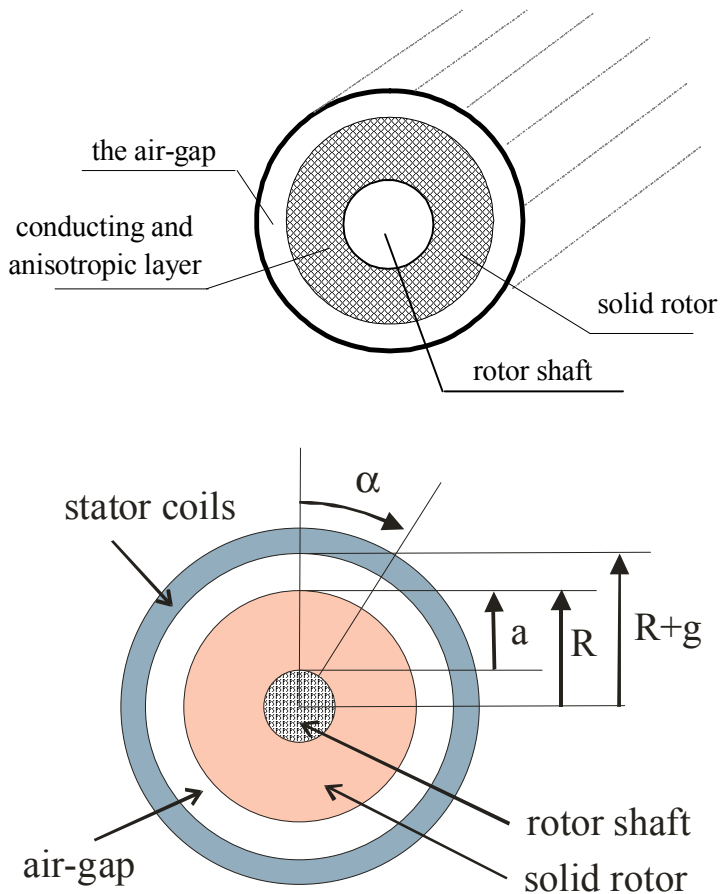

Fig. 4. Electromechanical converter with solid rotor a), its cross section b)

The numerical errors are omitted by applying analytical solution of electromagnetic field distribution and electromagnetic torque calculation.

The analysis of electromagnetic field has been provided with the help of variable separation method $[8,15]$. This way of analysis is chosen for giving precise insight into electromagnetic torque calculations, and may be treated as a benchmark test task $[12,13,14,15]$. For cylindrical converter $(\mathrm{r}, \alpha, \mathrm{z})$ the $\mathrm{z}$-component of magnetic vector potential is

$$
\vec{A}=\vec{A}_{z}=A_{z} \vec{i}_{z}=A \vec{i}_{z}
$$

The accuracy of such assumptions for magnetic vector potential symmetry results from simplified construction of mechanical converter - Fig.4. The magnetic flux density by means of vector magnetic potential is as follows

$$
\vec{B}=\frac{\vec{i}_{r}}{r} \frac{\partial A}{\partial \alpha}-\vec{i}_{\alpha} \frac{\partial A}{\partial r}
$$

where for cylindrical coordinate system $\left(\mathrm{x}_{1}=\mathrm{r}, \mathrm{x}_{2}=\alpha, \mathrm{x}_{3}=\right.$ $z)$. The model of induction motor with solid rotor can be analyzed under the following assumptions:

- electric displacement current vanishes (due to the small supply frequency),
- stator windings exert the sinusoidal p pair-pole $\mathrm{mmf}$

$$
\Theta_{\mathrm{s}}(\alpha)=\Theta_{\mathrm{s}} \cos \left(\mathrm{p} \alpha-2 \pi \mathrm{ft}+\alpha_{\mathrm{o}}\right)
$$

where $\Theta_{\mathrm{s}}$ stands for the magnitude of mmf, $\alpha$ is the position angle, f means the stator current frequency, there exists the cylindrical anisotropy of the magnetic reluctivities for machine rotor, thus the reluctivity matrix is built of four coefficients $v_{\mathrm{rr}}=v_{\mathrm{r}}, \quad v_{\alpha \alpha}=v_{\alpha}, \quad v_{\mathrm{r} \alpha}, \quad v_{\alpha \mathrm{r}}$, and all of them can be different, conductivity of rotor is $\gamma$ (isotropic parameter), rotor is homogeneous, hysteresis phenomenon does not appear.

The assumed symmetry of the electromechanical converter enables to carry two dimensional analysis. Magnetic field strength components are

$$
\begin{aligned}
& H_{r}=v_{r r} B_{r}+v_{r \alpha} B_{\alpha} \\
& H_{\alpha}=v_{\alpha r} B_{r}+v_{\alpha \alpha} B_{\alpha}
\end{aligned}
$$

the electric field strength is of the form of

$$
\vec{E}=-i \omega \vec{A}
$$

where A is magnetic field vector potential for steady-state, $\omega$ rotor current angular speed, $\vec{E}$ means electric field strength. Basing on Maxwell's equation

$$
\operatorname{curl}(\vec{H})=\not \vec{E}
$$

the Eqns (16) - (19) lead to equation

$$
\frac{v_{\alpha}}{r} \frac{\partial}{\partial r}\left(r \frac{\partial A}{\partial r}\right)-\frac{v_{r \alpha}+v_{\alpha r}}{r^{2}} \frac{\partial^{2} A}{\partial r \partial \alpha}+\frac{v_{r}}{r^{2}} \frac{\partial^{2} A}{\partial \alpha^{2}}=i \omega \gamma A
$$

The Eqn (20) is solved by the separation of the variables in the following form

$$
A=Z(\mathrm{r}) S(\alpha)=Z S,
$$

with the steady-state function $S(\alpha)$ for monoharmonic field in the form of:

$$
S(\alpha)=\exp (-i p \alpha)
$$

The variable separation for functions $\mathrm{Z}(\mathrm{r})$ and $\mathrm{S}(\alpha)$ leads to the differential equation

$$
\frac{d^{2} Z}{d r^{2}}+\frac{[1-2 c]}{r} \frac{d Z}{d r}-\left[\frac{v_{r} p^{2}}{v_{\alpha} r^{2}}+\beta^{2}\right] Z=0
$$

where $c=-i \frac{v_{r \alpha}+v_{\alpha r}}{2 v_{\alpha}}, \quad p_{B}=\sqrt{c^{2}+p^{2} v_{r r} / v_{\alpha \alpha}}$ with solution given in Table 1 .

TABLE I

SOLUTION OF THE DIFFERENTIAL EQUATIONS

\begin{tabular}{|c|c|}
\hline anisotropic rotor & the gap \\
\hline $\mathrm{Z}(\mathrm{z})=\mathrm{a}_{\mathrm{a}} \mathrm{f}_{1}(\mathrm{z})+\mathrm{b}_{\mathrm{a}} \mathrm{f}_{2}(\mathrm{z})$ & $\mathrm{Z}(\mathrm{z})=\mathrm{a}_{\delta} \mathrm{f}_{1}(\mathrm{z})+\mathrm{b}_{\delta} \mathrm{f}_{2}(\mathrm{z})$ \\
$\mathrm{f}_{1}(\mathrm{z})=\mathrm{z}^{\mathrm{c}} \mathrm{I}_{\mathrm{p}_{\mathrm{B}}}(\mathrm{z})$ & $\mathrm{f}_{1}(\mathrm{z})=\mathrm{z}^{\mathrm{c}+\mathrm{p}_{\mathrm{B}}}$, \\
$\mathrm{f}_{2}(\mathrm{z})=\mathrm{z}^{\mathrm{c}} \mathrm{K}_{\mathrm{p}_{\mathrm{B}}}(\mathrm{z})$ & $\mathrm{f}_{2}(\mathrm{z})=\mathrm{z}^{\mathrm{c}-\mathrm{p}_{\mathrm{B}}}$ \\
\hline
\end{tabular}

The four unknown constants $a_{a}, b_{a}, a_{\delta}, b_{\delta}$ can be evaluated by formulating the boundary conditions gap. The magnetic flux density components are equal to

$$
\left\{\begin{array}{c}
B_{a r}=\frac{\mathrm{p}}{\mathrm{r}}\left\{\mathrm{a}_{\mathrm{a}} f_{1}(r)+\mathrm{b}_{\mathrm{a}} f_{2}(\mathrm{r})\right\} \exp \left(-i p \alpha-i \frac{\pi}{2}\right) \\
B_{a \alpha}=-\frac{\mathrm{d}}{\mathrm{dr}}\left\{\mathrm{a}_{\mathrm{a}} f_{1}(r)+\mathrm{b}_{\mathrm{a}} f_{2}(r)\right\} \exp (-i p \alpha)
\end{array}\right.
$$

The magnetic field distribution leads to electromagnetic torque values. The electromagnetic torque $T_{\mathrm{e}}$ equals to

$$
T_{e}=\int_{\partial V} r H_{\alpha} B_{r} d S
$$


The total electromagnetic torque can be calculated also by means coenergy $\mathrm{W}_{\mathrm{c}}$ as follows

$$
T_{e}=\left.\frac{\partial W_{C}}{\partial \alpha}\right|_{j=c o n s t}=\int_{V}\left(\vec{j} \frac{\partial \vec{A}}{\partial \alpha}+\vec{B} \frac{\partial \vec{H}}{\partial \alpha}\right) d V
$$

The both Maxwell and coenergy methods give the same results always. The material (anisotropy) torque component is equal to

$$
T_{e M}=\int_{V} \frac{1}{2}\left(v_{r \alpha}-v_{\alpha r}\right) B_{r} \frac{\partial B_{\alpha}}{r \partial \alpha} d V
$$

and it does not vanish for asymmetrical anisotropy medium $v_{\mathrm{r} \alpha} \neq v_{\alpha \mathrm{r}}$.

Exemplary, it is considered cylindrical motor for it $\gamma=$ $5 \cdot 10^{6} \mathrm{~S} / \mathrm{m}$ (rotor conductivity), a $=0.05 \mathrm{~m}$ (conductive rotor layer width), $\mathrm{R}=0.1 \mathrm{~m}$ (rotor outer radius), $1=0.25 \mathrm{~m}$ (rotor length), $\mathrm{g}=0.0015 \mathrm{~m}$ (the gap width), $\Theta_{1}=150 \mathrm{~A}$ (magnetomotive force first harmonic), $p=2$ (pair pole number), $\mathrm{n}=20 \mathrm{~s}^{-1}$ (rotor speed), $\mathrm{v}_{\mathrm{rr}}=\mathrm{v}_{\mathrm{r}}=\mathrm{v}_{0} / 3$ (radial reluctivity), $v_{\alpha \alpha}=v_{\alpha}=v_{0} / 2$ (tangential reluctivity) and different anisotropy reluctivities $v_{\mathrm{r \alpha},} v_{\alpha \mathrm{r}}$ (Table 2).

The Table 2 and the Figs 5a,b confirm that if condition (4) is satisfied the first theorem thesis is fulfilled. However the cases c) and d) show that if condition (4) is not satisfied the first theorem thesis can not be applied.

TABLE II

EXAMPLES FOR TORQUES EVALUATION FOR THE FIRST THEOREM PRESENTATION

\begin{tabular}{|l|ll|c|}
\hline & \multicolumn{2}{|c|}{ Magnetic reluctivities } & The first theorem \\
\hline a) & $v_{\mathrm{r} \alpha}=0.0 v_{0}$ & $v_{\alpha \mathrm{r}}=0.0 v_{0}$ & Eqn (4) is true - Fig 5a. \\
\hline b) & $v_{\mathrm{r} \alpha}=0.2 v_{0}$ & $v_{\alpha \mathrm{r}}=0.2 v_{0}$ & Eqn (4) is true - Fig 5b \\
\hline c) & $v_{\mathrm{r} \alpha}=0.5 v_{0}$ & $v_{\alpha \mathrm{r}}=0$ & Eqn (4) is false - Fig 5c \\
\hline d) & $v_{\mathrm{r} \alpha}=0$ & $v_{\alpha \mathrm{r}}=0.5 v_{0}$ & Eqn (4) is false - Fig 5d \\
\hline
\end{tabular}

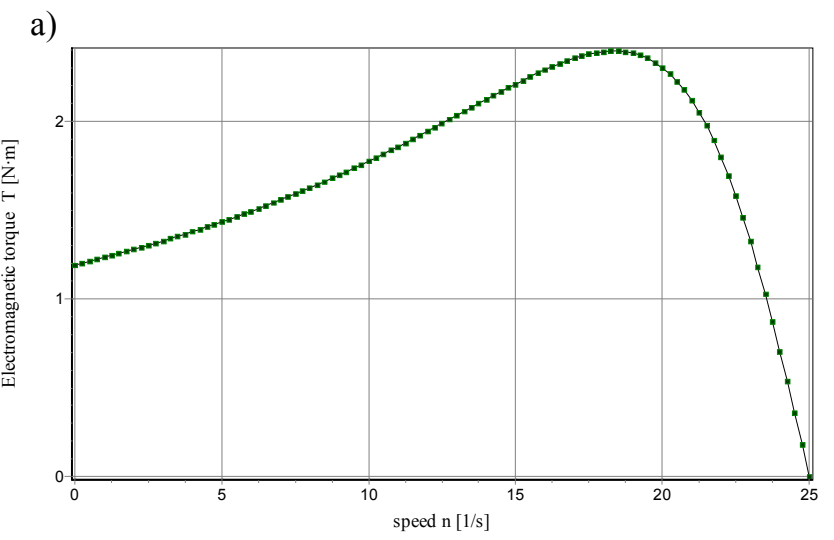

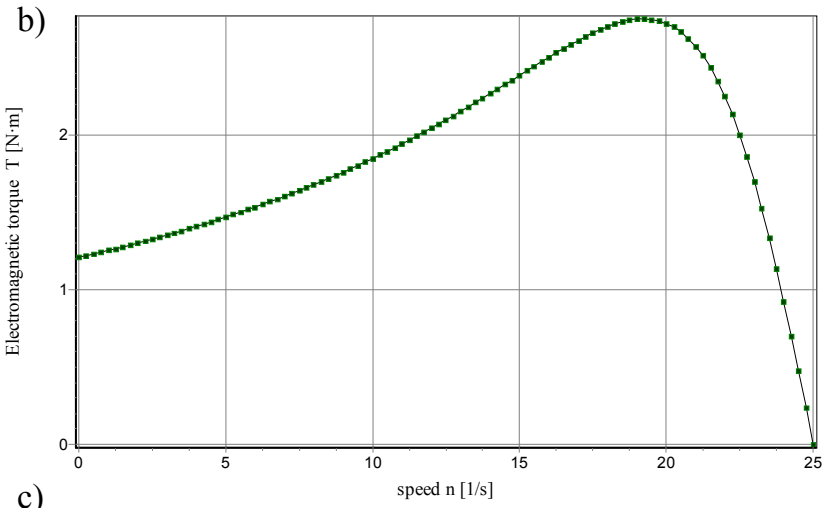

c)

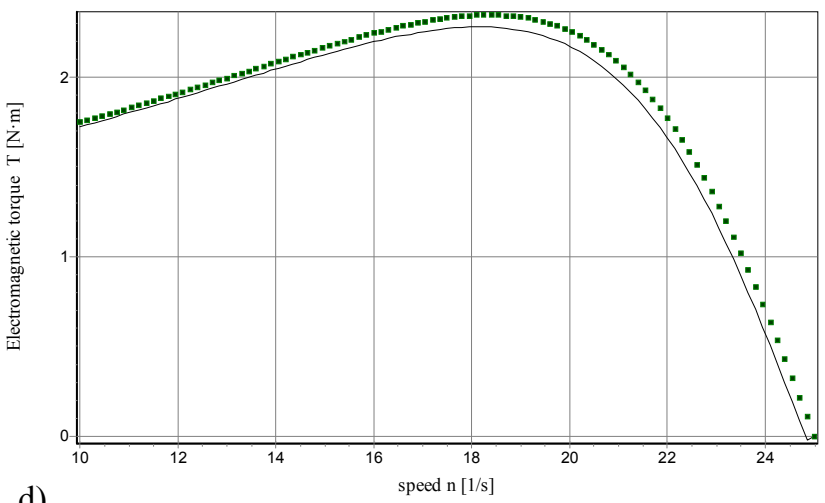

d)

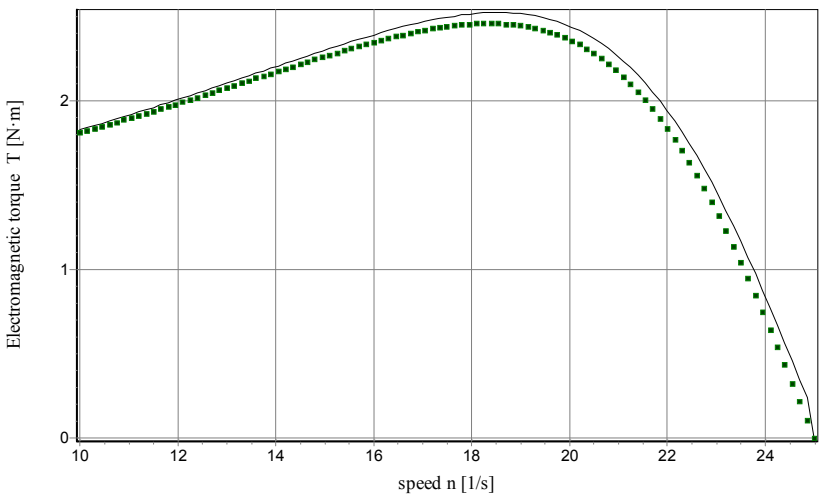

Fig. 5. Electromagnetic torque vs. speed (Maxwell method - line, Lorentz method - points)

Examples for the second theorem are presented for the same data of cylindrical solid rotor motor and for introduced the anisotropic magnetically gap as specified in the Table 3.

TABLE III

EXAMPLES FOR TORQUES EVALUATION FOR THE SECOND THEOREM PRESENTATION

\begin{tabular}{|l|c|c|}
\hline & Reluctivities & The second theorem \\
\hline a) & $v_{\mathrm{r} \alpha \delta}=0.0 \mathrm{v}_{0}$ & Eqn (12) IS SATISFIED \\
& $v_{\alpha \mathrm{\alpha r} \delta}=0.0 \mathrm{v}_{0}$ & - Fig 6a \\
\hline b) & $v_{\mathrm{r} \alpha \delta}=0.5 \mathrm{v}_{0}$ & Eqn (12) IS NOT SATISFIED \\
& $v_{\alpha \mathrm{\alpha} \delta}=0.0 \mathrm{v}_{0}$ & - Fig 6b \\
\hline
\end{tabular}




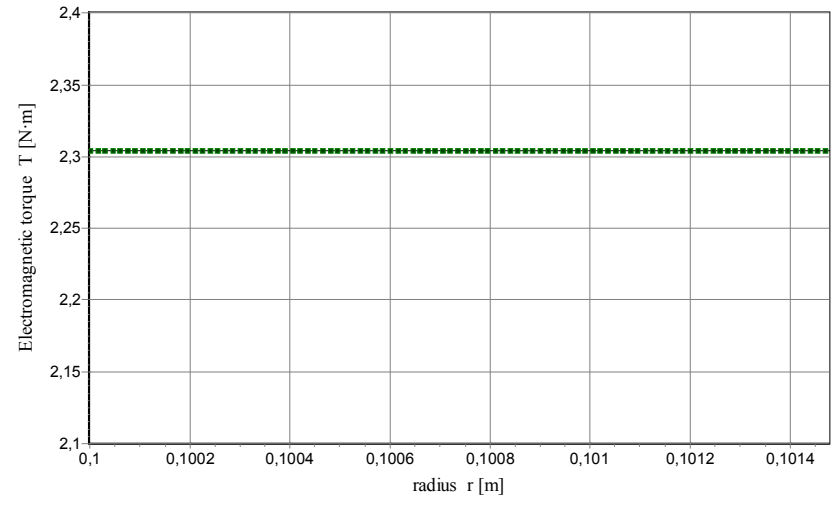

b)

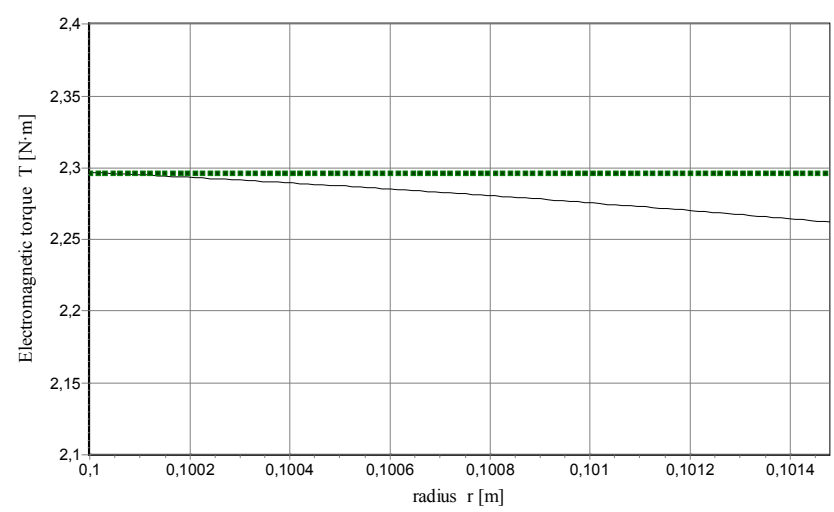

Fig. 6. Electromagnetic torque vs. radius for the gap region (Maxwell method - line, Lorentz method - points)

The Figs 6a,b present the electromagnetic torque value obtained by Maxwell's method for different radius of integration surface (cylindrical surface). The radius changes from $\mathrm{r}=\mathrm{R}$ (outer solid rotor surface) up to $\mathrm{R}+\mathrm{g}$ (inner stator surface). The Table 3 and the Fig 6 a confirm that if condition (12) is satisfied the second theorem thesis is fulfilled. In opposite, in the case b) is shown that if condition (12) is not satisfied the second theorem thesis can not be applied.

\section{CONCLUSIONS}

There have been presented two theorems stated in the main text about electromagnetic force/torque in anisotropic medium are proved by author in 1997 and 2007.

The first theorem

for magnetic field where the following conditions are satisfied

- no nonhomogeneous force(no reluctance force),

- no hysteresis phenomenon appears,

the force calculated by Lorentz's (volume integral) and Maxwell (surface integral) methods lead to the same result for magnetically anisotropic region if reluctivity matrix is symmetrical i.e. for $u \neq v$ it is satisfied $v_{v u}=v_{u v}$

The second theorem

The electromagnetic force/ torque value calculated with the help of

either volume integral or Maxwell stress tensor surface integral

does not depend on surface position in the gap

if the condition (12) is satisfied.

In order to confirm the theoretical results described above, an example of electromechanical converter with solid conductive rotor it was considered.

\section{REFERENCES}

[1] Landau L.D., Lifszyc E.M.: The classical theory of fields, Pergamon, New York, 1951

[2] Jackson J.D.: Classical electrodynamics, John Wiley, New York, 1999

[3] Benda O.: Torque exerted on anisotropic magnetic medium by electromagnetic wave, IEEE Transaction On Magnetics, 1969, 5, (4), pp. 921-924

[4] Henrotte F.: Handbook for the computation of electromagnetic forces in a continuous medium, Newsletter of the International Compumag Society, ISSN 1026-0854, Vol. 11, No. 2, (2004)

[5] Fujioka T.: A new anisotropic correction to the formula of force and torque on materials, Proceedings of International Symposium on Applied Electromagnetics and Mechanics ISEM, Tokyo, 23-27 (2002)

[6] Henrotte F., Vande Sande H., Deliége G., Hameyer K.: Electromagnetic force density in a ferromagnetic material, IEEE Transaction on Magnetics, 40(2), 553-556, (2004)

[7] Adkins B., Harley P.G.: The general theory of alternating current machines, Chapman and Hall, London, 1978

[8] Binns K.J., Lawrenson P.J., Trowbridge C.W.: The analytical and numerical solution of electric and magnetic fields, John Wiley \& Sons, 1992

[9] Engelmann S., Nethe A., Scholz T., Stahlmann H-D.: Application of force enhencement with ferrofluids in linear stepping motor model. Conference Proceedings ISTET 2003, vol. II pp.425-428, Warsaw

[10] Nethe A., Scholz T., Stahlmann H-D.: An analytical solution method for magnetic fields using the Fourier analysis and its application of ferrofluid driven electric machines, Proc. ISTET 2003, Warsaw, Poland, Vol. II, pp. 421-424 (2003)

[11] Arkkio A.: Time stepping finite element analysis of induction motors. Proceedings of International Conference on Electrical Machines ICEM'88, pp.275-280, Pisa, 1988.

[12] D. Spałek D.: Theorem about electromagnetic force surface representation in anisotropic region. Journal of Technical Physics, Vol. XLVIII No. 3-4,pp. 135-145, 2007

[13] Spałek D.: Analytical electromagnetic field and forces calculation for linear, cylindrical and spherical electromechanical converters, Bulletin of the Polish Academy of Sciences. Technical Sciences, 52, (3), pp. 239-250, 2004

[14] Spałek D.: Spherical induction motor with anisotropic rotor analytical solutions for electromagnetic field distribution, electromagnetic torques and power losses, International Compumag Society. Testing Electromagnetic Analysis Methods (T.E.A.M.) problem no. $34 \mathrm{http}: / /$ www.compumag.co.uk/team.html, 2007

\section{APPENDIX}

Lorentz's force density in the well-known form of

$$
\vec{f}_{L}=\rho \vec{E}+\vec{j} \times \vec{B}
$$

can be presented with the help of Maxwell's equations

$$
\operatorname{curl} \vec{H}=\vec{j}+\frac{\partial \vec{D}}{\partial t}, \quad \rho=\operatorname{div} \overrightarrow{\mathrm{D}}
$$

as follows:

$$
\overrightarrow{\mathrm{f}}_{\mathrm{L}}=\operatorname{curl} \overrightarrow{\mathrm{H}} \times \overrightarrow{\mathrm{B}}+\overrightarrow{\mathrm{H}} \operatorname{div} \overrightarrow{\mathrm{B}}-\frac{\partial(\overrightarrow{\mathrm{D}} \times \overrightarrow{\mathrm{B}})}{\partial \mathrm{t}}+\overrightarrow{\mathrm{D}} \times \operatorname{curl} \overrightarrow{\mathrm{E}}+\overrightarrow{\mathrm{E}} \operatorname{div} \overrightarrow{\mathrm{D}}
$$

where it was taken into account that $\vec{H} \cdot \operatorname{div} \vec{B}=0$.

Let us present constitutive relation in the following general form:

$$
H_{u}=v_{u w} B_{w}-\Delta I_{u}
$$

where reluctivities $v_{\text {uw }}$ could be asymmetrical, $\Delta \mathrm{I}_{\mathrm{u}}$ is the $\mathrm{u}^{\text {th }}$ component of magnetic polarisation vector (it follows form the hysteresis phenomenon). The first and second components on the right-hand side of (A4) can be written in the form of

$$
\operatorname{curl} \overrightarrow{\mathrm{H}} \times \overrightarrow{\mathrm{B}}+\overrightarrow{\mathrm{H}} \operatorname{div} \overrightarrow{\mathrm{B}}=\overrightarrow{\mathrm{i}}_{\mathrm{u}} \operatorname{div}_{\mathrm{u}}\left(\vec{\sigma}_{\mu \mathrm{u}}\right)-\vec{\Delta}_{\mu}-\overrightarrow{\mathrm{N}}_{\mu}-\overrightarrow{\mathrm{Q}}_{\mu}-\overrightarrow{\mathrm{M}}_{\mu}
$$
where it has been denoted (no summation due to $|\mathrm{u}|$ ):

$$
\begin{gathered}
\operatorname{div}_{u}(*)=\mathrm{L}_{|\mathrm{u}|}^{-1} \operatorname{div}\left(L_{u} \cdot(*)\right) \\
\vec{\sigma}_{\mu u}=-H_{u} \vec{B}+\vec{i}_{u} e_{\mu}, \quad e_{\mu}=\frac{1}{2} \vec{H} \vec{B}
\end{gathered}
$$

and 


$$
\vec{N}_{\mu}=\frac{1}{2} B_{u} B_{w} \operatorname{grad}\left(v_{u w}\right)
$$

which is called the nonhomogenous force component, and

$$
\overrightarrow{\mathrm{Q}}_{\mu}=\frac{1}{2} \operatorname{grad}\left(\mathrm{B}_{\mathrm{u}} \Delta \mathrm{I}_{\mathrm{u}}\right)-\mathrm{B}_{\mathrm{u}} \operatorname{grad}\left(\Delta \mathrm{I}_{\mathrm{u}}\right)
$$

which is called the hysteresis component (resulting from hysteresis phenomenon), and an auxiliary vector defined as follows:

$$
\overrightarrow{\mathrm{M}}_{\mu}=\frac{1}{2}\left(\mathrm{v}_{\mathrm{vu}}-\mathrm{v}_{\mathrm{uv}}\right) \mathrm{B}_{\mathrm{v}} \operatorname{grad}\left(\mathrm{B}_{\mathrm{u}}\right)
$$

which is called anisotropy component, and an additional auxiliary vector defined as follows:

$$
\vec{\Delta}_{\mu}=\frac{1}{2} B_{v} H_{v} \vec{i}_{u} \frac{\partial \ln \left(L_{v}^{2}|L|^{-1}\right)}{L_{u} \partial x_{u}}
$$

for orthogonal curvilinear co-ordinate system $\mathrm{u}, \mathrm{v}, \mathrm{w}$.

The fourth and fifth components on the right-hand side of (A4) can be rearranged in the same manner

$$
\operatorname{curl} \vec{E} \times \vec{D}+\vec{E} \operatorname{div} \vec{D}=\vec{i}_{u} \operatorname{div}_{u}\left(\vec{\sigma}_{\varepsilon u}\right)-\vec{\Delta}_{\varepsilon}-\vec{N}_{\varepsilon}-\vec{Q}_{\varepsilon}-\vec{M}_{\varepsilon}
$$

where the constitutive relation is introduced as follows:

$$
\mathrm{D}_{\mathrm{u}}=\varepsilon_{\mathrm{uw}} \mathrm{E}_{\mathrm{w}}-\Delta \mathrm{P}_{\mathrm{u}}
$$

where $\Delta \mathrm{P}_{\mathrm{u}}$ is the $\mathrm{u}^{\text {th }}$ component of electric polarisation vector, and

$$
\begin{gathered}
\vec{\sigma}_{\varepsilon u}=-E_{u} \vec{D}+\vec{i}_{u} e_{\varepsilon}, \quad e_{\varepsilon}=\frac{1}{2} \vec{E} \vec{D} \\
\vec{N}_{\varepsilon}=-\frac{1}{2} E_{u} E_{w} \operatorname{grad}\left(\varepsilon_{\text {uw }}\right) \\
\vec{Q}_{\varepsilon}=\frac{1}{2} \operatorname{grad}\left(\Delta P_{u} E_{u}\right)-E_{u} \operatorname{grad}\left(\Delta P_{u}\right) \\
\vec{M}_{\varepsilon}=-\frac{1}{2}\left(\varepsilon_{v u}-\varepsilon_{u v}\right) E_{v} \operatorname{grad}\left(E_{u}\right)
\end{gathered}
$$

and an auxiliary vector

$$
\vec{\Delta}_{\varepsilon}=\frac{1}{2} D_{v} E_{v} \vec{i}_{u} \frac{\partial \ln \left(L_{v}^{2}|L|^{-1}\right)}{L_{u} \partial x_{u}}
$$

Hence $\mathrm{Eq}(\mathrm{A} 4)$ takes the form

$$
\vec{f}_{L}=-\frac{\partial(\vec{D} \times \vec{B})}{\partial t}-\vec{i}_{u} \operatorname{div}_{u}\left(\vec{\sigma}_{u}\right)-\vec{\Delta}-\vec{N}-\vec{Q}-\vec{M}
$$

where

$$
\begin{gathered}
\vec{\sigma}_{u}=-E_{u} \vec{D}-H_{u} \vec{B}+\vec{i}_{u} e \\
e=e_{\varepsilon}+e_{\mu}=\frac{1}{2} \vec{E} \vec{D}+\frac{1}{2} \vec{H} \vec{B} \\
\vec{N}=\vec{N}_{\varepsilon}+\vec{N}_{\mu}=-\frac{1}{2} E_{u} E_{w} \operatorname{grad}\left(\varepsilon_{\mathrm{uv}}\right)+\frac{1}{2} B_{u} B_{w} \operatorname{grad}\left(v_{\mathrm{uv}}\right) \\
\vec{Q}=\vec{Q}_{\varepsilon}+\vec{Q}_{\mu} \\
\vec{M}=\frac{1}{2}\left(v_{\mathrm{vu}}-v_{\mathrm{uv}}\right) B_{v} \operatorname{grad}\left(\mathrm{B}_{\mathrm{u}}\right) \\
-\frac{1}{2}\left(\varepsilon_{\mathrm{vu}}-\varepsilon_{\mathrm{uv}}\right) E_{v} \operatorname{grad}\left(\mathrm{E}_{\mathrm{u}}\right) \\
\vec{\Delta}=\left(\frac{1}{2} D_{v} E_{v}+\frac{1}{2} B_{v} H_{v}\right) \vec{i}_{u} \frac{\partial \ln \left(\mathrm{L}_{\mathrm{v}}^{2}|\mathrm{~L}|^{-1}\right)}{L_{u} \partial x_{u}}
\end{gathered}
$$

The Maxwell's force density $\vec{f}$ is equal to

$$
\vec{f}=\vec{f}_{L}+\vec{f}_{P}+\vec{N}+\vec{Q}+\vec{M}=-\vec{i}_{u} d i v_{u}\left(\vec{\sigma}_{u}\right)-\vec{\Delta}
$$

where Maxwell's stress tensor equals

$$
\vec{\sigma}_{u}=-H_{u} \vec{B}-E_{u} \vec{D}+\vec{i}_{u} e
$$

and the electromagnetic field impulse force density [4]

$$
\vec{f}_{P}=\frac{\partial(\vec{D} \times \vec{B})}{\partial t} .
$$

Equation (A10) is valid for orthogonal curvilinear coordinate system. The tensor notation is more compact than the presented above vector notation, but the presented vector notation it is more convenient for devices analysis. The residual component $\vec{\Delta}$ given by (A9) appears when the vector notation presented above is used. Such a component does not appear for tensor notation of (A10). It should be emphasized that the operator $\operatorname{div}_{\mathrm{u}}()$ in (A10) differs from the well-known operator $\operatorname{div}()$ and it is useful for surface representation of total electromagnetic torque as shown below.

The main theoretical problem presented in this paper is to consider the equivalence of volume electromagnetic forces by certain surface integral (the so-called surface representation problem, Maxwell's stress tensor representation). It is well-known [1] that the surface integral representation for electromagnetic field forces can be introduced for electromagnetic field regions where the stress tensor is symmetrical (for isotropic media). It is also stated [1] that symmetry of stress tensor is necessary and sufficient condition for the surface representation. In this paragraph it is proved that

for electromagnetic field where the following conditions are satisfied

- impulse force component $\vec{f}_{P}=0$ (low field frequency),

- nonhomogeneous force $\vec{N}=0$ (no reluctance force), and

- hysteresis component $\vec{Q}=0$ (no hysteresis phenomenon), the both Lorentz's and Maxwell's methods do not lead to the same result for anisotropic region if either reluctivity or permittivity matrix is asymmetrical i.e. for $\mathrm{u} \neq \mathrm{v}$

$$
v_{v u} \neq v_{u v} \quad \varepsilon_{u v} \neq \varepsilon_{v u}
$$

In other words, the symmetry of Maxwell stress tensor is not a sufficient condition for the surface representation. Moreover, for media with symmetric reluctivity and permittivity matrices the Lorentz's force can be presented by surface-integral of the stress tensor.

The mathematical proof of this theorem is based on Eq. (A10). Namely, using the assumptions specified above, Eq. (A10) takes the form of

$$
\vec{f}=\vec{f}_{L}+\vec{M}
$$

According to Eqs (A8) and (A12), the total force density (given by divergence) is not equal to Lorentz force density for some anisotropic regions i.e. for media with asymmetric reluctivity matrix ( $\vec{\Delta}=0$ for Cartesian co-ordinate system). In other words, the surface-integral representation (after applying Gaussian theorem) is possible for media with symmetric both reluctivity and permittivity matrices.

The above statement, however it applies only to a small range group of anisotropic materials, is important from theoretical point of view.

\section{BIOGRAPHY}

Dariusz Spalek was born in Poland, on October 23, 1963. He graduated from the Silesian University of Technology in 1988 (doctoral thesis 1994, habilitation thesis 2002). He works at Institute of Electrical Engineering and Informatics of Silesian University of Technology in Gliwice, Poland. 